\title{
Impacto da Atividade Inflamatória e Uso de Glicocorticóide nas Variáveis Nutricionais da Artrite Idiopática Juvenil(*)
}

\section{Impact of Inflammatory Activity and Glucocorticoid Use in the Nutritional Variables of Juvenile Idiopathic Arthritis}

\author{
Letícia Souza Bisotto $^{(1)}$, Ricardo Machado Xavier ${ }^{(2)}$, Sandra Helena Machado ${ }^{(3)}$, \\ Markus Bredemeier ${ }^{(4)}$, João Carlos Tavares Brenol ${ }^{(5)}$
}

\section{RESUMO}

Objetivo: avaliar o estado nutricional na artrite idiopática juvenil (AIJ), a influência da atividade inflamatória e o uso de glicocorticóide. Métodos: foram estudados 116 pacientes com AIJ diagnosticados segundo os critérios da ILAR/1997. Subtipo e atividade da doença foram determinados por reumatologistas pediátricos, e a dose cumulativa de glicocorticóide foi revisada nos prontuários dos pacientes. Foram determinados os percentis do índice de massa corporal (IMC) e da prega cutânea tricipital (PCT) e o escore Z da estatura, de acordo com a OMS. Considerou-se baixo peso e baixa adiposidade quando o IMC e a PCT apresentavam-se abaixo do percentil 5. Baixa estatura foi definida por escore $Z$ de estatura para idade menor do que -2 . O nível sérico de IGF-1 foi medido por meio de radioimunoensaio. Resultados: as prevalências de baixo peso, baixa adiposidade e baixa estatura foram $16,4 \%, 20,7 \%$ e 10,4\%, respectivamente. Níveis séricos reduzidos de IGF-1 foram observados em 14 pacientes $(12,1 \%)$. Os fatores negativamente associados com o escore Z da estatura na análise de regressão multivariável foram: duração da doença (coeficiente parcial de correlação, intervalo de confiança de 95\%: $-0,370,-0,527$ a $-0,188: \mathrm{p}<0,001)$, velocidade de hemossedimentação (VHS) $(-0,357,-0,516$ a -0,174; p<0,001), subtipo da doença sistêmico ou poliarticular $(-0,290,-0,459$ a $0,100 ; p=0,003)$, enquanto não houve associação significante com dose cumulativa de glicocorticóide $(0,086,-0,111$ a 0,277: $\mathrm{p}=0,391)$. Nenhuma dessas variáveis foi significativamente associada com os percentis de IMC e PCT, porém, pacientes com os subtipos sistêmico e poliarticular tenderam a apresentar percentis de IMC mais baixos

\begin{abstract}
Objective: To assess the nutritional status in juvenile idiopathic arthritis (JIA) and the influence of inflammatory activity and glucocorticoid use. Methods: One hundred and sixteen patients were evaluated. Disease subtype and disease activity were defined by the attending physician, and the cumulative glucocorticoid dose was recorded by chart review. The percentiles of body mass index (BMI) and triceps skinfold (TSF) and the $Z$-score for height were determined: low weight and low adiposity were diagnosed when BMI and TSF were below the 5 th percentile. Short stature was defined by a $Z$-score of height for age $<-2$. The serum level of IGF-I was measured by radioimmunoassay. Results: The prevalences of low weight, low adiposity and short stature were $16.4 \%, 20.7 \%$ and $10.4 \%$, respectively. Low IGF-1 serum level was found in 14 patients (12.1\%). The factors negatively associated with the $Z$-score of height in multivariable regression analysis were disease duration (partial correlation coefficient, 95\% confidence interval: -0.370 , -0.527 to $-0.188 ; p<0.001)$, erythrocyte sedimentation rate (ESR) (-0.357, -0.516 to $-0.174 ; p<0.001)$, polyarticular or systemic subtype $(-0.290,-0.459$ to $-0.100 ; p=0.003)$, while there was no significant association with the cumulative dose of glucocorticoids (0.086, -0.111 to 0.277; $p=0.391$ ). None of these variables were significantly associated with the percentiles of BMI or TSF, but patients with a systemic or polyarticular subtype tended to present lower percentiles of $B M I(p=0.051)$. Conclusions: Nutritional status is frequently
\end{abstract}

\footnotetext{
* Trabalho realizado no Serviço de Reumatologia do Hospital de Clínicas de Porto Alegre, Universidade Federal do Rio Grande do Sul (UFRGS), Porto Alegre, RS, Brasil, com apoio do Fundo de Auxílio à Pesquisa e Ensino em Reumatologia da Sociedade Brasileira de Reumatologia. Este estudo foi parcialmente financiado pelo Fundo de Incentivo à Pesquisa do Hospital de Clínicas de Porto Alegre (FIPE/HCPA). Recebido em 20/04/2005. Aprovado, após revisão, em 02/10/2005.

1. Nutricionista, mestra em Ciências Médicas, UFRGS

2. Professor adjunto, Faculdade de Medicina da UFRGS, Serviço de Reumatologia do HCPA.

3. Médica pediatra, doutoranda em Pediatria, UFRGS

4. Médico reumatologista, mestre em Ciências Médicas, UFRGS.

5. Professor adjunto, Faculdade de Medicina, UFRGS, chefe do Serviço de Reumatologia, HCPA.

Endereço para correspondência: Dr. Ricardo M. Xavier. Serviço de Reumatologia do Hospital de Clínicas de Porto Alegre (HCPA), Rua Ramiro Barcellos, 2.350, sala 645, Bairro Rio Branco, CEP 90035-003. Porto Alegre (RS), Brasil. E-mail: rmaxavier@hcpa.ufrgs.br
} 
$(p=0,051)$. Conclusões: pacientes com AIJ apresentam freqüentemente comprometimento do estado nutricional. A duração e subtipo da doença e o VSG são fatores independentemente associados à baixa estatura. A dose cumulativa de glicocorticóide não esteve associada de maneira independente com a baixa estatura ou a outras variáveis nutricionais.

Palavras-chave: artrite idiopática juvenil, parâmetros nutricionais, baixa estatura, fator de crescimento similar à insulina (IGF-1).

\section{INTRODUÇÃO}

Artrite idiopática juvenil (AIJ) é a doença reumática inflamatória crônica mais comum da infância ${ }^{(1)}$. Freqüentemente tem sido relatado comprometimento do estado nutricional, avaliado por meio de medidas antropométricas e bioquímicas, na AIJ. Entre as anormalidades das variáveis antropométricas observadas na AIJ, estudos prévios sugerem que a prevalência de baixo peso situa-se entre $4 \%$ e $46,6 \%{ }^{(2-5)}$ e a prevalência de baixa estatura encontra-se entre $10 \%$ e $41 \%{ }^{(4,6-12)}$. As possíveis causas implicadas nessas alterações são anorexia $^{(2)}$, restrições alimentares ${ }^{(13)}$, má absorção dos nutrientes ${ }^{(14)}$, aumento do catabolismo e do requerimento energético $^{(15)}$, limitação de atividade física ${ }^{(16)}$, redução de absorção de vitamina $\mathrm{D}^{(17)}$, redução de secreção de hormônio do crescimento ${ }^{(6)}$ e tratamento medicamentoso com glicocorticóides $^{(7)}$.

Entre as anormalidades dos marcadores bioquímicos do estado nutricional, baixos níveis de albumina, ferro e IGF-1 são freqüentemente observados na $\mathrm{AIJ}^{(4)}$. $\mathrm{O}$ nível sérico de IGF-1 (insulin-like growth factor-I), agente efetor do hormônio do crescimento e considerado o melhor marcador de balanço nitrogenado ${ }^{(18)}$, freqüentemente encontra-se reduzido, estando associado à baixa estatura na $\mathrm{AIJ}^{(4,6,19)}$.

Diversas características clínicas têm sido correlacionadas com o comprometimento nutricional: duração e atividade da doença ${ }^{(4,6,8,12,15)}$, redução da ingestão dietética ${ }^{(2)}$, subtipo sistêmico ou poliarticular de doença ${ }^{(4,5,8,12,20)}$ e uso de glicocorticóide. No entanto, há divergências sobre a associação do uso de glicocorticóide e sua dosagem com a redução da estatura, havendo estudos sugerindo uma associação relevante $\mathrm{e}^{(4,7,12,19,21-22)}$ e outros estudos sem demonstrar $\operatorname{associação~}^{(5-6,8,20)}$.

Diante desses dados divergentes na literatura, nosso objetivo foi estudar a prevalência do estado nutricional comprometido em um grupo de pacientes com AIJ. Também objetivou-se estudar a influência da atividade compromised in JIA. The duration and subtype of the disease and the ESR are factors independently associated with short stature. The cumulative dose of glucocorticoids was not independently associated with short stature or other nutricional variables.

Keywords: juvenile idiopathic arthritis, nutritional parameters, short stature, insulin-like growth factor-1 (IGF-1).

inflamatória e o uso de glicocorticóide nas variáveis antropométricas e bioquímicas que refletem o estado nutricional.

\section{MATERIAL E MÉTODOS}

Pacientes. O estudo foi realizado no ambulatório de Reumatologia do Hospital de Clínicas de Porto Alegre (HCPA). Os pacientes deste estudo foram recrutados consecutivamente em três centros clínicos de atendimento de reumatologia pediátrica de Porto Alegre (RS). Cento e dezesseis pacientes ( 74 sexo feminino e 42 sexo masculino, com idade entre 2 e 20 anos incompletos), que satisfaziam os critérios diagnósticos de Durban/ILAR $1997^{(23)}$ e apresentavam tempo mínimo de diagnóstico de um ano, foram convidados a participar do estudo. Os critérios de exclusão foram a presença de outras doenças sistêmicas concomitantes à AIJ ou incapacidade de realização das medidas antropométricas.

Avaliação clínica. Após o recrutamento, os pacientes foram submetidos a uma entrevista padronizada avaliando as características da doença, uso de medicamentos, medidas antropométricas e renda familiar. A presença de atividade da doença foi determinada por meio de avaliação clínica por reumatologistas pediátricos. A doença foi considerada: a) ativa, havendo aumento no número de articulações acometidas apesar do uso de terapia medicamentosa; b) estável, havendo estabilidade no número de articulações acometidas com o uso de terapia medicamentosa; c) inativa, sem evidência de sinovite e/ou atividade extra-articular sem uso de terapia medicamentosa por menos do que dois anos; d) em remissão, sem evidência de sinovite e/ou atividade extra-articular sem uso de terapia medicamentosa por dois anos ou mais.

A dose cumulativa de glicocorticóide foi relatada como dose equivalente em miligramas de prednisona. O comprometimento da articulação temporomandibular (ATM) foi diagnosticado pela presença de sintomas associados e ava- 
liação radiológica (radiografia simples e tomografia computadorizada), quando necessário. Fator reumatóide (FR) foi determinado por nefelometria e a presença de fator antinuclear (FAN) foi testada por método de imunofluorescência indireta usando células HEp-2 como substrato.

Variáveis antropométricas. Os dados antropométricos foram mensurados de acordo com as normas da Organização Mundial da Saúde (OMS) e sempre pelo mesmo aferidor (LSB). O peso foi aferido em balança com graduação em 10 gramas, com os pacientes descalços e usando somente um avental padrão. A estatura foi mensurada com estadiômetro, com os pacientes descalços e com os pés juntos, os calcanhares contra a parede, e a altura lida foi arredondando para o 0,5 cm mais próximo (OMS, 1995) e o escore $Z$ foi calculado, considerando como padrão de referência o do National Center of Health Statistics $\mathrm{NCHS}^{(25)}$. De acordo com a OMS ${ }^{(24)}$, a estatura foi classificada em: baixa estatura moderada $(-3 \leq$ escore $Z<-2)$ e baixa estatura grave (escore $Z<-3$ ). O índice de massa corporal (IMC) foi calculado pela fórmula peso/estatura ${ }^{2}$ e utilizaram-se curvas de percentis de IMC para 2-20 anos de idade desenvolvidos pelo NCHS em colaboração com Centers for Disease Control and Prevention - CDC Growth Charts 2000(24,25). Os percentis obtidos dessa forma foram classificados de acordo com a escala da OMS (baixo peso, os valores abaixo do percentil 5 , eutrofia, entre os percentis 5 e 85 , e sobrepeso, acima do percentil 85)(24). A prega cutânea tricipital (PCT) foi medida na face posterior do braço não dominante, na metade da linha que liga o processo acromial ao olécrano. A aferição foi realizada três vezes consecutivas, sendo que o resultado obtido foi a média das aferições. Utilizou-se para aferição um Plicômetro Científico (Cescorf, Porto Alegre, RS, Brasil), com alta precisão e sensibilidade de 0,1 milímetro. Os resultados foram comparados com tabelas distribuídas em percentis ${ }^{(26)}$. Segundo a OMS ${ }^{(24)}$, os valores abaixo do percentil 5 representam baixa adiposidade; valores entre 5 e 90 representam normalidade; valores acima do percentil 90 representam alta adiposidade.

Variáveis bioquímicas. A coleta de sangue foi realizada no mesmo dia da avaliação clínica. Foram coletadas amostras de sangue venoso $(2-3 \mathrm{ml})$ por venopunção periférica de todos os pacientes para a dosagem dos testes laboratoriais de bioquímica básica, incluindo hemograma completo, velocidade de hemossedimentação (VHS), albumina e cálcio total. Alíquotas do soro, obtidas após centrifugação, foram congeladas a $-80^{\circ} \mathrm{C}$ até o ensaio para análise do IGF-1, o qual foi realizado em um só momento por meio de ensaio imunorradiométrico (IRMA) de dois sítios descritos por Miles et al. (IRMA Active IGF-1 DSL-5600, Texas/EUA) ${ }^{(27)}$. Esse ensaio inclui uma etapa de extração simples, na qual o IGF-1 é separado de suas proteínas de ligação no soro. Realizaram-se extração ácido/etanol, neutralização e análise em um único ensaio. Os níveis de IGF-1 são expressos em $\mathrm{ng} / \mathrm{ml}$, e o escore Z do IGF-1 para cada paciente foi determinado por meio dos valores de referência propostos pelo fabricante, que variam de acordo com a idade e sexo. Os níveis de hemoglobina foram considerados baixos em crianças quando menores do que $11 \mathrm{~g} / \mathrm{dl}$, e quando menores do que 12 e $13 \mathrm{~g} / \mathrm{dl}$ para adolescentes do sexo feminino e masculino, respectivamente. A contagem de linfócitos totais foi considerada baixa quando $<1500 / \mathrm{mm}^{3}$. No laboratório do HCPA, os valores normais para albumina sérica variam de 3,5-4,8 g/dL, e os valores normais do cálcio total, de $8,6-10 \mathrm{~g} / \mathrm{dL}$.

Análise estatística. Os dados foram analisados utilizando-se os programas EPI-INFO versão $6^{(28)}$ e SPSS para Windows versão $11.0^{(29)}$. Variáveis categóricas foram apresentadas como números e proporções. Variáveis quantitativas foram testadas graficamente (com curvas de probabilidade normal) e estatisticamente (com teste de goodnessof-fit de Kolmogorov-Smirnov) quanto à normalidade da distribuição. Variáveis com distribuição normal foram apresentadas como média e desvio-padrão, sendo que as comparações entre os grupos foram realizadas através do teste $t$ de Student ou análise de variância (ANOVA), com teste de Tukey para avaliação de contrastes. Variáveis quantitativas não-normais foram apresentadas como mediana e percentis (25 e 75) e as comparações entre grupos foram realizadas usando-se os testes de Mann-Whitney ou Kruskal-Wallis. Os coeficientes de correlação de Pearson $\left(r_{p}\right)$ e Spearman $\left(r_{s}\right)$, juntamente com seus intervalos de confiança de $95 \%$, foram usados para testar correlações envolvendo variáveis contínuas normais e não-normais, respectivamente. A associação entre variáveis categóricas foi testada usando-se o teste do qui-quadrado e o teste exato de Fisher. Valores de $\mathrm{P}<0,05$ (bicaudais) foram considerados estatisticamente significativos.

Para avaliação da associação de variáveis clínicas com o escore $\mathrm{Z}$ da estatura, foi elaborado um modelo de regressão linear múltipla. A seleção de variáveis independentes para o modelo foi baseada na capacidade da variável em representar algum aspecto específico da doença (não foram 
utilizados métodos automáticos para a seleção de variáveis). Os pressupostos do modelo de regressão foram avaliados por meio do teste de Kolmogorov-Smirnov para normalidade dos resíduos, do teste de White para heteroscedasticidade, da avaliação dos fatores de inflação de variância para detecção de colinearidade, e de testes para associação não-linear com o auxílio do programa Grett ${ }^{(30)}$. Coeficientes de correlação parcial ( $\mathrm{r}$ parcial) e intervalos de confiança de $95 \%$ foram estimados para as variáveis independentes incluídas no modelo.

Aspectos éticos. Foi obtido consentimento livre e esclarecido dos pacientes, pais ou responsáveis de cada paciente. O projeto foi aprovado pelo Comitê de Ética em Pesquisa do Hospital de Clínicas de Porto Alegre.

\section{RESULTADOS}

As características demográficas e clínicas dos pacientes estão descritas na Tabela 1. Observa-se predominância de pacientes caucasóides, do sexo feminino e com a forma poliarticular de AIJ na amostra. Pacientes com as formas poliarticular e sistêmica apresentaram maior prevalência de uso corrente e maior dose cumulativa de glicocorticóide.

A Tabela 2 apresenta uma descrição das características antropométricas (classificadas de acordo com critérios da
OMS) na amostra. As prevalências gerais de baixo peso e baixa adiposidade identificadas pelo IMC e pelo PCT foram $16,4 \%$ e $20,7 \%$, respectivamente. Pacientes com a forma poliarticular apresentaram a maior prevalência de baixo peso pelo IMC $(24,5 \%)$. Baixa estatura esteve presente em $10,4 \%$ dos pacientes, sendo mais prevalente na forma poliarticular da doença.

A comparação dos parâmetros bioquímicos de acordo com o subtipo da doença encontra-se na Tabela 3. Observou-se maior prevalência de anemia e linfopenia na forma poliarticular de doença, assim como níveis de cálcio reduzidos nesse subtipo. Pacientes com a forma sistêmica apresentaram, de modo geral, valores laboratoriais semelhantes à forma poliarticular. Nenhum paciente apresentou baixos níveis de albumina sérica.

A Tabela 4 compara as características antropométricas apresentadas pelos diferentes subtipos da doença. A forma poliarticular da doença apresentou o maior deficit de estatura entre todos os grupos. Não houve diferenças quanto aos percentis de IMC e PCT entre os grupos. No entanto, comparando o grupo composto pelos subtipos sistêmico e poliarticular com o grupo composto pelos subtipos oligoarticular e psoriásico, o primeiro grupo citado tende a apresentar percentis de IMC mais baixos (mediana [percentis $25 ; 75], 37,5[9,5 ; 80,0]$ versus $60,0[20,0 ; 95,0]$, respectivamente; $\mathrm{p}=0,051)$.

TABELA 1

CARACTERÍSTICAS DEMOGRÁFICAS E CLÍNICAS DOS PACIENTES SEGUNDO O SUBTIPO DA DOENÇA

\begin{tabular}{|c|c|c|c|c|c|}
\hline & Oligoarticular $(n=48)$ & Poliarticular $(n=53)$ & Sistêmica $(n=12)$ & Psoriásica $(n=3)$ & Total $(n=116)$ \\
\hline Sexo feminino $-\mathrm{n}(\%)$ & $30(62,5)$ & $36(67,9)$ & $5(41,7)$ & $3(100)$ & $74(63,8)$ \\
\hline Caucasianos - n (\%) & $42(87,5)$ & $46(86,8)$ & $11(91,7)$ & $2(66,7)$ & $101(87,1)$ \\
\hline Idade - média \pm DP & $10,1 \pm 4,1$ & $11,7 \pm 4,1$ & $11,0 \pm 3,7$ & $12,4 \pm 6,5$ & $11,0 \pm 4,1$ \\
\hline $\begin{array}{l}\text { Início da doença (idade) - } \\
\text { mediana (percentis } 25 ; 75 \text { ) }\end{array}$ & $4,0(2,0 ; 7,4)$ & $5,0(2,5 ; 8,0)$ & $4,5(3,6 ; 6,7)$ & $10,0(4,0 ; 11,0)$ & $5,0(2,2 ; 8,0)$ \\
\hline Doença Ativa - n (\%) & $24(50,0)$ & $35(66,0)$ & $5(41,7)$ & $2(66,7)$ & $66(56,9)$ \\
\hline $\mathrm{FR} \geq 20 \mathrm{ng} / \mathrm{ml}-\mathrm{n}(\%)$ & $1(2,1)$ & $4(7,5)$ & $1(8,3)$ & $0(0,0)$ & $6(5,2)$ \\
\hline FAN $\geq 1: 80-n(\%)$ & $6(12,5)$ & $6(11,3)$ & $0(0,0)$ & $0(0,0)$ & $12(10,3)$ \\
\hline $\begin{array}{l}\text { Duração da doença em anos - } \\
\text { mediana (percentis } 25 ; 75 \text { ) }\end{array}$ & $4,5(3,0 ; 6,6)$ & $5,4(2,6 ; 9,1)$ & $4,7(2,3 ; 8,2)$ & $3,7(1,3 ; 8,1)$ & $4,7(2,7 ; 8,3)$ \\
\hline Corticoterapia atual - n (\%) & $14(29,2)$ & $32(60,4)$ & $7(58,3)$ & $1(33,3)$ & $54(46,6)$ \\
\hline $\begin{array}{l}\text { Dose cumulativa de } \\
\text { glicocorticóide }(\mathrm{mg})^{\star}- \\
\text { mediana (percentis } 25 ; 75 \text { ) }\end{array}$ & $400(0,0 ; 1800)$ & $1800(630 ; 3150)$ & $1687,5(281 ; 4342,5)$ & $900(0,0 ; 1725)$ & $1420(70,0 ; 2657,5)$ \\
\hline
\end{tabular}

* Equivalente à dose de prednisona; FR: fator reumatóide; FAN: fator antinuclear 
TABELA 2

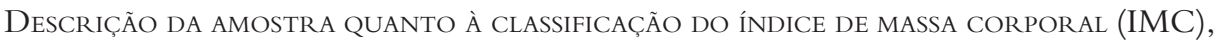
prega cutânea tricipital (PCT) e da estatura segundo a OrganizaÇão Mundial da Saúde (OMS)ネ

\begin{tabular}{|c|c|c|c|c|c|}
\hline & $\begin{array}{l}\text { Oligoarticular } \\
\qquad(n=48)\end{array}$ & $\begin{array}{l}\text { Poliarticular } \\
\quad(n=53)\end{array}$ & $\begin{array}{l}\text { Sistêmica } \\
(\mathrm{n}=12)\end{array}$ & $\begin{array}{l}\text { Psoriásica } \\
\quad(n=3)\end{array}$ & $\begin{array}{c}\text { Total } \\
(n=116)\end{array}$ \\
\hline \multicolumn{6}{|l|}{ Classificação do IMC } \\
\hline Baixo Peso & $5(10,4)$ & $13(24,5)$ & $1(8,3)$ & $0(0,0)$ & $19(16,4)$ \\
\hline Eutrofia & $26(54,2)$ & $31(58,5)$ & $10(83,3)$ & $3(100)$ & $70(60,3)$ \\
\hline Sobrepeso & $17(35,4)$ & $9(17,0)$ & $1(8,3)$ & $0(0,0)$ & $27(23,3)$ \\
\hline \multicolumn{6}{|l|}{ Classificação do PCT } \\
\hline Baixa Adiposidade & $8(16,7)$ & $12(22,6)$ & $3(25,0)$ & $1(33,3)$ & $24(20,7)$ \\
\hline Eutrofia & $32(66,7)$ & $35(66,0)$ & $9(75,0)$ & $2(66,7)$ & $78(67,2)$ \\
\hline Excessiva adiposidade & $8(16,7)$ & $6(11,3)$ & $0(0,0)$ & $0(0,0)$ & $14(12,1)$ \\
\hline \multicolumn{6}{|l|}{ Classificação da estatura } \\
\hline Normalidade & $47(97,9)$ & $44(83,0)$ & $10(83,3)$ & $3(100)$ & $104(89,6)$ \\
\hline Baixa estatura moderada & $1(2,1)$ & $5(9,4)$ & $0(0,0)$ & $0(0,0)$ & $6(5,2)$ \\
\hline Baixa estatura grave & $0(0,0)$ & $4(7,6)$ & $2(16,7)$ & $0(0,0)$ & $6(5,2)$ \\
\hline
\end{tabular}

* Os valores representam número e porcentual. 0s porcentuais são calculados sobre o total de pacientes de cada subtipo da doença

TABELA 3

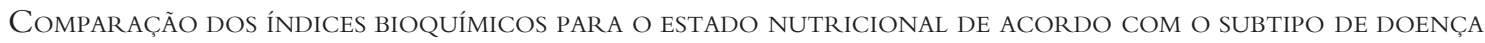

\begin{tabular}{|c|c|c|c|c|c|c|}
\hline & $\begin{array}{l}\text { Oligoarticular } \\
\qquad(n=48)\end{array}$ & $\begin{array}{l}\text { Poliarticular } \\
\quad(n=53)\end{array}$ & $\begin{array}{l}\text { Sistêmica } \\
(n=12)\end{array}$ & $\begin{array}{l}\text { Psoriásica } \\
(n=03)\end{array}$ & $\begin{array}{c}\text { Total } \\
(n=116)\end{array}$ & $\mathbf{P}^{*}$ \\
\hline Anemia - n (\%) & $8(16,7)$ & $22(41,5)$ & $5(41,7)$ & $1(33,3)$ & $36(31,0)$ & 0,027 \\
\hline Linfopenia - n (\%) & $1(2,1)$ & $9(17,0)$ & $1(8,3)$ & $0(0,0)$ & $11(9,5)$ & 0,070 \\
\hline $\begin{array}{l}\text { Albumina }(\mathrm{g} / \mathrm{dl})- \\
\text { média } \pm \mathrm{DP}\end{array}$ & $4,5 \pm 0,3$ & $4,5 \pm 0,3$ & $4,4 \pm 0,3$ & $4,7 \pm 0,2$ & $4,5 \pm 0,3$ & 0,161 \\
\hline $\begin{array}{l}\text { Cálcio total }(\mathrm{mg} / \mathrm{dl}) \\
\text { - média } \pm \mathrm{DP} \dagger\end{array}$ & $9,6 \pm 0,5^{A}$ & $9,3 \pm 0,5^{\mathrm{B}}$ & $9,2 \pm 0,5^{\mathrm{AB}}$ & $9,4 \pm 0,4^{\mathrm{AB}}$ & $9,4 \pm 0,5$ & 0,039 \\
\hline $\begin{array}{l}\text { Níveis reduzidos de } \\
\text { IGF-1 }(\mathrm{ng} / \mathrm{ml})-\mathrm{n}(\%)\end{array}$ & $4(8,3)$ & $7(13,2)$ & $3(25,0)$ & $0(0,0)$ & $14(12,1)$ & 0,382 \\
\hline
\end{tabular}

* ANOVA, qui-quadrado ou teste exato de Fisher; † As letras em sobrescrito representam grupos estatisticamente diferentes $(P<0,05)$ identificados pelo teste de Tukey

TABELA 4

Comparação dos escores Z da estatura, percentis do IMC e Percentis do PCT de acordo COM o SUbtipo da doenÇA

\begin{tabular}{|c|c|c|c|c|c|c|}
\hline & $\begin{array}{l}\text { Oligoarticular } \\
\qquad(n=48)\end{array}$ & $\begin{array}{l}\text { Poliarticular } \\
\quad(n=53)\end{array}$ & $\begin{array}{l}\text { Sistêmica } \\
(n=12)\end{array}$ & $\begin{array}{l}\text { Psoriásica } \\
(n=03)\end{array}$ & $\begin{array}{c}\text { Total } \\
(n=116)\end{array}$ & $P^{*}$ \\
\hline $\begin{array}{l}\text { Escore } Z \text { da estatura - } \\
\text { média } \pm \mathrm{DP} \dagger\end{array}$ & $0,57 \pm 1,13^{A}$ & $-0,80 \pm 1,70^{B}$ & $-0,51 \pm 1,98^{\mathrm{AB}}$ & $-0,05 \pm 0,21^{\mathrm{AB}}$ & $-0,18 \pm 1,62$ & $<0,001$ \\
\hline $\begin{array}{l}\text { Percentil do IMC - } \\
\text { mediana (percentis } 25 ; 75 \text { ) }\end{array}$ & $51,0(18,7 ; 95,0)$ & $37,5(7,5 ; 80,0)$ & $41,2(28,5 ; 81,0)$ & $80,0(62,5 ; 80,0)$ & $47,5(17,5 ; 83,5)$ & 0,205 \\
\hline $\begin{array}{l}\text { Percentil da PCT - } \\
\text { mediana (percentis } 25 ; 75 \text { ) }\end{array}$ & $17,5(7,5 ; 68,7)$ & $17,5(5,0 ; 62,5)$ & $25,0(4,0 ; 68,7)$ & $37,5(3,0 ; 80,0)$ & $18,7(5,0 ; 62,5)$ & 0,937 \\
\hline
\end{tabular}


A Tabela 5 avalia as correlações entre características clínicas da doença e as variáveis antropométricas aferidas. $\mathrm{O}$ VSG, a duração da doença e a dose cumulativa de glicocorticóide foram significativamente associados com a redução da estatura na análise bivariada. Nenhuma das variáveis testadas foi significativamente correlacionada com os percentis de IMC e PCT.

Pacientes com doença clinicamente ativa tiveram menores escores $Z$ da estatura em comparação com pacientes com a doença controlada ou inativa (média [DP], -0,53 $[1,79], \mathrm{n}=66$ versus $0,28[1,25], \mathrm{n}=50$, respectivamente; $\mathrm{p}=0,007)$. Contudo, não houve diferenças significantes entre pacientes com doença ativa ou controlada/inativa nos percentis de IMC (mediana [percentis 25; 75], 50,0 $[17,5 ; 85,0]$ versus $45,0[17,5 ; 82,0]$, respectivamente; $\mathrm{p}=0,788)$ e nos percentis de $\operatorname{PCT}(17,5[5,0 ; 70,0]$ versus $20,0[7,5 ; 62,5]$, respectivamente; $\mathrm{p}=0,825)$.

No presente estudo, somente um paciente apresentou envolvimento da articulação temporomandibular (ATM), revelando-se comprometimento das variáveis antropométricas e bioquímicas no mesmo. Os resultados das classificações antropométricas desse paciente foram baixo peso para IMC, baixa adiposidade para PCT e baixa estatura com escore $\mathrm{Z}$ menor que $-5 \mathrm{DP}$. Entre os parâmetros laboratoriais observaram-se anemia e linfopenia.

Foi criado um modelo de regressão linear múltipla tendo como variável dependente o escore $\mathrm{Z}$ da estatura. As variáveis consideradas possivelmente relevantes para determinar a variável dependente foram incluídas no modelo. A variável dose cumulativa de glicocorticóide teve de ser transformada em forma logarítmica por causa de um caso que apresentou um valor extremamente elevado. O modelo final está apresentado na Tabela 6. A duração da doença, VHS e os subtipos sistêmico e poliarticular foram associados independentemente e aditivamente com a redução da estatura dos pacientes, enquanto que a estatura materna foi positivamente associada com um maior escore $\mathrm{Z}$ da estatura.
A dose cumulativa de glicocorticóide não foi preditora independente na redução dos escores da estatura.

Quatorze pacientes (12,1\% do total de casos, 7 com subtipo poliarticular da doença, 4 na forma oligoarticular e 3 no subtipo sistêmico) apresentaram níveis reduzidos de IGF-1. $\mathrm{O}$ escore $\mathrm{Z}$ dos níveis de IGF-1 foi positivamente correlacionado com o escore $Z$ da estatura ( $r_{p}$, IC 95\%: 0,236, $0,056$ a 0,$401 ; p=0,011)$. No entanto, um modelo de regressão incluindo também um termo quadrático e cúbico para o escore Z do IGF-1 demonstrou melhor associação com o escore Z da estatura (r múltiplo, IC 95\%: 0,379, 0,210 a 0,$526 ; p=0,001)$, sugerindo a possibilidade de uma associação não-linear entre estas duas variáveis. A duração da doença ( $r_{s}$, IC 95\%: -0,051, -0,231 a 0,133; p=0,587) e a dose cumulativa de glicocorticóide $(-0,124,-0,299$ a 0,060; $\mathrm{p}=0,185)$ não foram significativamente correlacionadas com o escore Z do IGF-1. Pacientes com os subtipos sistêmico ou poliarticular da doença apresentaram escores Z do IGF-1 similares aos dos subtipos oligoarticular e psoriásico (média [DP], -0,78 [1,21] versus -0,80 [1,09], respectivamente; $\mathrm{p}=0,921)$. No entanto, houve uma correlação negativa entre VHS e escore $\mathrm{Z}$ do IGF-1 ( $\mathrm{r}_{\mathrm{S}}$, IC 95\%: -0,345, -0,5000 a $0,170, p<0,001)$, e pacientes com doença clinicamente ativa apresentaram escores $Z$ de IGF-1 mais baixos do que os pacientes com doença controlada/inativa (média [DP], -0,98 $[1,06]$ versus $-0,54[1,23]$, respectivamente; $p=0,039)$. Em um modelo de regressão linear multivariável tendo como variável dependente o escore Z do IGF-1 (mas incluindo as mesmas variáveis independentes apresentadas na Tabela 6), a única variável independente significativamente associada com o escore Z do IGF-1 foi o VHS (r parcial, IC 95\%: 0,422, -0,570 a -2,247; $\mathrm{p}<0,001)$. Neste modelo, a dose cumulativa de glicocorticóide não foi significativamente associada com baixos escores de IGF-1 (r parcial, IC 95\%: 0,056, -0,141 a 0,249; $\mathrm{p}=0,573)$. Não houve associações significativas dos escores Z do IGF-1 com os percentis de IMC $(p=0,932)$ ou com os percentis de PCT $(p=0,450)$.

TABELA 5

CORRELAÇÕES NÃO-PARAmÉtriCAS dAS VARiÁveis CLÍNICAS COM O ESCORE Z DA ESTATURA E COM OS PERCENTIS DE IMC E PCT

\begin{tabular}{|c|c|c|c|c|c|c|}
\hline & $\begin{array}{l}\text { Escore } Z \text { da estatura } \\
\qquad(\text { IC } 95 \%)^{*}\end{array}$ & $\mathrm{p}$ & $\begin{array}{l}\text { Percentil de IMC } \\
\quad(\text { IC } 95 \%)^{*}\end{array}$ & $\mathbf{P}$ & $\begin{array}{l}\text { Percentil de PCT } \\
\text { IC } 95 \%)^{*}\end{array}$ & $\mathrm{p}$ \\
\hline $\begin{array}{l}\text { Dose cumulativa de } \\
\text { glicocorticóide }(n=116)\end{array}$ & $-0,23(-0,40$ a $-0,05)$ & 0,013 & $-0,05(-0,23$ a 0,13$)$ & 0,585 & $-0,08(-0,26$ a 0,10$)$ & 0,371 \\
\hline Duração da doença $(n=116)$ & $-0,39(-0,54 \mathrm{a}-0,23)$ & $<0,001$ & $-0,11(-0,29$ a 0,07$)$ & 0,221 & $-0,02(-0,20$ a 0,16$)$ & 0,818 \\
\hline $\begin{array}{l}\text { Velocidade de } \\
\text { hemossedimentação }(n=111) \dagger\end{array}$ & $-0,47(-0,60$ a $-0,31)$ & $<0,001$ & $-0,04(-0,22$ a 0,15$)$ & 0,697 & $0,02(-0,17$ a 0.20$)$ & 0,869 \\
\hline
\end{tabular}

* Coeficiente de correlação de Spearman e intervalo de confiança de 95\%; † Cinco casos foram excluídos por falta dos dados 
TABela 6

MODELO DE REGRESSÃo LiNEAR MULTIVARIÁVEL UTILIZANDO O ESCORE Z DA ESTATURA DOS PACIENTES COMO VARIÁVEL DEPENDENTE.

\begin{tabular}{|c|c|c|c|}
\hline Variáveis independentes & $B^{*}$ & Coeficiente de correlação parcial (IC $95 \%$ ) $\dagger$ & $\mathrm{p}$ \\
\hline Duração da doença, anos & $-0,134$ & $-0,370(-0,527$ a $-0,188)$ & $<0,001$ \\
\hline Velocidade de hemossedimentação, $\mathrm{mm} / \mathrm{h}$ & $-0,019$ & $-0,357(-0,516$ a $-0,174)$ & $<0,001$ \\
\hline Subtipo poliarticular ou sistêmico $\ddagger$ & $-0,783$ & $-0,290(-0,459$ a $-0,100)$ & 0,003 \\
\hline Dose cumulativa de glicocorticóide $\left(\log _{10}\left[\right.\right.$ dose $\left.\left._{(\mathrm{mg})}+1\right]\right)$ & 0,078 & $0,086(-0,111$ a 0,277$)$ & 0,391 \\
\hline Doença ativa $\ddagger$ & $-0,340$ & $-0,133(-0,320$ a 0,064$)$ & 0,183 \\
\hline Estatura materna, $\mathrm{cm} \S$ & 0,076 & $0,398(0,220$ a 0,551$)$ & $<0,001$ \\
\hline Constante वा & $-10,675$ & - & $<0,001$ \\
\hline
\end{tabular}

A associação entre a renda familiar e as variáveis nutricionais (escore $\mathrm{Z}$ da estatura, percentis do IMC e do PCT) e laboratoriais (anemia e calcemia) foi testada, sendo que não se observou nenhuma associação estatisticamente significativa (não apresentado).

\section{DISCUSSÃO}

O presente estudo avaliou as variáveis nutricionais, antropométricas e bioquímicas em uma população de pacientes com AIJ, bem como se buscou identificar a relação desses parâmetros com as características clínicas da doença.

Vários estudos demonstraram comprometimento das variáveis nutricionais na AIJ. Entre as variáveis antropométricas, a baixa estatura é freqüentemente observada e pode implicar problemas psicológicos relacionados à auto-estima dos pacientes. Observamos uma prevalência de baixa estatura de $10,4 \%$. A prevalência de baixa estatura apresentada na literatura varia de $10 \%$ a $41 \%{ }^{(4,6-12)}$, e os subtipos mais freqüentemente acometidos são o sistêmico e o poliarticular. A grande variação de prevalência de baixa estatura na literatura pode ser explicada pela seleção de pacientes e diferentes métodos de classificação para baixa estatura empregados nos diversos estudos. Simon et al. ${ }^{(7)}$ observaram prevalência de $41 \%$ de baixa estatura final, porém estudaram somente pacientes com o subtipo sistêmico de doença que estivessem em tratamento com glicocorticóide por longo período de tempo. Alta prevalência de baixa estatura $(38,8 \%)$ também foi identificada em outro estudo ${ }^{(10)}$, em que o critério para classificação de baixa estatura foi um índice estatura/idade abaixo do percentil 20, enquanto a classificação da OMS considera baixa estatura somente se abaixo do percentil 3. Henderson et al. ${ }^{(11)}$ observaram prevalência de baixa estatura (classificada como valores abaixo do percentil 5) de $19 \%$. Allen et al. ${ }^{(6)}$, que utilizaram o escore $Z$ na avaliação da estatura (o mesmo usado no presente estudo), relataram prevalência de baixa estatura de 26\%. Entretanto, esses autores consideraram baixa estatura um escore $Z<-1,5 \mathrm{DP}$, diferentemente do critério recomendado pela OMS e empregado no presente estudo $(<-2,0$ DP $)$. No estudo brasileiro ${ }^{(9)}$, que conta com 41 pacientes com as formas oligoarticular e poliarticular da doença, a prevalência de baixa estatura foi de 19,5\%, usando critérios idênticos aos empregados em nosso estudo. As prevalências de baixa estatura nos outros estudos com critérios semelhantes de seleção e de classificação de pacientes variaram entre $10 \%$ e $15 \%{ }^{(4,8,12)}$.

No presente estudo, os subtipos sistêmico e poliarticular, a duração da doença e o VHS associaram-se significativa e independentemente à redução na estatura. Estudos prévios observaram resultados similares em relação ao subtipo ${ }^{(4,5,8,12,20)}$, duração ${ }^{(4,6,8,12)}$, atividade e severidade da doença( ${ }^{(4,12)}$. Considerando-se que os subtipos sistêmico e poliarticular apresentam quadro inflamatório mais severo ${ }^{(2,4,6,9)}$, esses achados confirmam a importância da severidade e persistência do quadro inflamatório para o deficit de crescimento.

Existem controvérsias a respeito da associação entre corticoterapia e baixa estatura. Alguns estudos observacionais associaram o uso, duração ou dose cumulativa de glicocorticóide com redução na estatura ${ }^{(4,7,12,19,21,22)}$. A maioria desses estudos não utilizou análise multivariável para ajustar para o efeito de possíveis fatores de viés, como 
subtipo, duração e severidade da doençça ${ }^{(7,19,21,22)}$. Zak et al..$^{(12)}$ e Garcia-Consuegra Molina et al. ${ }^{(4)}$ apresentaram modelos de regressão linear múltipla em que o uso de glicocorticóides foi considerado um fator independente para a redução da estatura ou baixa velocidade de crescimento. Os modelos finais apresentados por aqueles autores não levaram em consideração a possível influência de variáveis como subtipo e duração de doença, que foram excluídas pelos métodos stepwise de seleção de variáveis (métodos automáticos realizados pelos programas estatísticos). Esses métodos de seleção de variáveis podem ter baixo poder para detectar fatores de confusão, particularmente quando operando em amostras pequenas ${ }^{(31)}$ e o conjunto de variáveis selecionadas pode ser difícil de reproduzir em outras amostras retiradas da mesma população ${ }^{(32)}$. De qualquer modo, dada a natureza observacional dos estudos, pode ser difícil discriminar os efeitos dos glicocorticóides e da severidade da doença na estatura, considerando-se que os glicocorticóides são freqüentemente usados para controlar a atividade inflamatória resistente aos antiinflamatórios nãoesteróides e a drogas anti-reumáticas de ação lenta.

Por outro lado, um número semelhante de estudos ${ }^{(5,6,8,20)}$, contando também com amostras pequenas (máximo 67 pacientes), não demonstraram associações entre corticoterapia e baixa estatura. Esses resultados negativos podem ser relacionados ao baixo poder estatístico para detectar efeitos clinicamente relevantes. No presente estudo, que conta com uma amostra relativamente grande de pacientes, observou-se uma correlação negativa (estatisticamente significativa) entre a dose cumulativa de glicocorticóide e o escore Z da estatura na análise bivariada (Tabela 5). No entanto, após um adequado ajuste para importantes variáveis de viés (duração e subtipo de doença, VHS) na regressão linear múltipla, não se observou uma associação independente entre corticoterapia e baixa estatura. Tendo-se em mente as limitações de estudos transversais, o intervalo de confiança de $95 \%$ do coeficiente parcial de correlação $(-0,111$ a 0,277 , Tabela 6) torna improvável que um efeito negativo clinicamente relevante dos glicocorticóides na estatura não tenha sido detectado nesse estudo. Uma conclusão mais definitiva do efeito independente dos corticóides na estatura pode somente ser possível com estudos de coorte bem controlados ou ensaios clínicos randomizados.

A prevalência de baixo peso no presente estudo (16,4\%) está dentro da variação de valores apresentados em estudos prévios $^{(2-5)}$ e está particularmente próxima aos resultados apresentados por Lofthouse et al. ${ }^{(5)}$, que utilizaram critérios de classificação semelhantes aos do presente estudo. A associação do peso e adiposidade com as características clínicas da doença também tem sido objeto de estudo. Lofthouse et al. ${ }^{(5)}$ observaram IMC mais baixo e mais baixo PCT em pacientes com o subtipo poliarticular em comparação com um grupo de controles normais. Haugen et al. ${ }^{(22)}$ estudando 220 adultos com AIJ observaram IMC similar em pacientes com doença ativa, inativa e controles normais, mas os pacientes apresentaram menor PCT do que os controles normais. Cleary et al. ${ }^{(3)}$ observaram IMC mais baixo em pacientes com inflamação articular persistente e número mais elevado de juntas com perda de amplitude de movimento. No presente estudo, não se observou uma associação da duração da doença, VHS e da atividade da doença com os percentis do IMC e da PCT. Entretanto, os subtipos sistêmico e poliarticular tenderam a apresentar percentis mais baixos de IMC, sugerindo uma possível associação entre o estado inflamatório e baixo peso.

A dose cumulativa de glicocorticóide não foi significativamente associada com os percentis do IMC e da PCT. Resultados similares foram obtidos por Allen et al.$^{(6)} \mathrm{e} \mathrm{Liem}$ et al. ${ }^{(8)}$, que não encontraram uma associação entre uso de corticóide e peso. Uma possível explicação para os resultados negativos é que o aumento do apetite e acumulação de gordura subcutânea promovidos pelo glicocorticóide podem ser contrabalançados pelo catabolismo promovido pela atividade de doença na AIJ.

O IGF-1, anteriormente conhecido como somatomedina C, é o principal agente efetor do hormônio do crescimento no osso e outros tecidos ${ }^{(18)}$. Níveis reduzidos de IGF-1 têm sido observados em $9 \%$ a $35 \%$ dos pacientes com $\mathrm{AJJ}^{(4,6,19)}$. $\mathrm{Na}$ presente amostra níveis reduzidos de IGF-1 ocorreram em 14 pacientes $(12,1 \%)$ e baixa estatura estava presente em 4 desses pacientes. Como previamente observado ${ }^{(4,6,19)}$, níveis reduzidos de IGF-1 foram associados com escores de estatura mais baixos. Garcia-Consuegra Molina et al..$^{(4)}$ observaram correlações negativas significativas (em análise bivariada) da duração de doença ativa e da dose cumulativa de glicocorticóide com os escores de IGF-1. No presente estudo, o VHS e a atividade da doença foram as variáveis clínicas associadas com níveis mais baixos de IGF-1. Esses resultados corroboram a conclusão de que é a persistente atividade inflamatória a responsável pela baixa estatura observada na AIJ, enquanto que o uso de glicocorticóide pode ter no máximo um efeito deletério pequeno no crescimento.

Existe evidência de que o processo inflamatório e suas conseqüências nutricionais na AIJ poderiam ser causadas 
principalmente pelos efeitos de citocinas como o TNF- $\alpha$ e as interleucinas 1 e $6^{(15)}$. Essas citocinas promovem um estado de acidose metabólica, a atividade aumentada de osteoclastos e a inibição da secreção do hormônio do crescimento, favorecendo o hipercatabolismo e o impacto no crescimento linear. Nossas observações de maior deficit de crescimento em pacientes com atividade inflamatória mais elevada corrobora a idéia da participação de citocinas pró-inflamatórias nessa complicação. Essas observações são relevantes, considerando a emergência de novas terapias que inibem a ação dessas citocinas.

A restrição alimentar secundária ao envolvimento da ATM e da saúde oral poderiam ser consideradas algumas das possíveis causas de envolvimento nutricional na $\mathrm{AIJ}^{(13,33,34)}$. Entretanto, não há estudos avaliando associação entre o envolvimento da ATM e o estado nutricional de pacientes com AIJ. Na presente amostra, a disfunção de ATM foi claramente mostrada em um único paciente, sugerindo que esse fator tem uma relevância limitada no comprometimento nutricional na AIJ.

Por causa dos problemas nutricionais e o aumento do catabolismo e do gasto de energia associados com inflamação ${ }^{(2,13-15)}$, é possível que um trabalho de reabilitação nutricional possa ser benéfico para esses pacientes, ajudando

\section{REFERÊNCIAS}

1. Lang BA, Shore A: A review of current concepts on the pathogenesis of juvenile rheumatoid arthritis. J Rheumatol 21 Suppl: 1-15, 1990.

2. Haugen MA, Hoyeraal HM, Larsen S, Gilboe IM, Trygg K: Nutrient intake and nutritional status in children with juvenile chronic arthritis. Scand J Rheumatol 21: 165-70, 1992.

3. Cleary AG, Lancaster GA, Annan F, Sills JA, Davidson JE: Nutritional impairment in juvenile idiopathic arthritis. Rheumatology (Oxford) 43: 1569-73, 2004

4. Garcia-Consuegra Molina J, Merino Munoz R, Lama More R, Coya Vina J, Gracia Bouthelier R: Crecimiento en ninos con artritis idiopatica juvenil [Growth in children with juvenile idiopathic arthritis]. An Pediatr (Barc) 58: 529-37, 2003.

5. Lofthouse CM, Azad F, Baildam EM, Akobeng AK: Measuring the nutritional status of children with juvenile idiopathic arthritis using the bioelectrical impedance method. Rheumatology (Oxford) 41: 1172-7, 2002.

6. Allen RC, Jimenez M, Cowell CT: Insulin-like growth factor and growth hormone secretion in juvenile chronic arthritis. Ann Rheum Dis 50: 602-6, 1991.

7. Simon D, Fernando C, Czernichow P, Prieur AM: Linear growth and final height in patients with systemic juvenile idiopathic arthritis treated with longterm glucocorticoids.J Rheumatol 29: 1296-300, 2002.

8. Liem JJ, Rosenberg AM: Growth patterns in juvenile rheumatoid arthritis. Clin Exp Rheumatol 21: 663-8, 2003. a manter o desenvolvimento físico normal. Por exemplo, uma dieta hipercalórica durante as fases mais ativas da doença poderia melhorar o estado de excessivo catabolismo, potencialmente reduzindo as perdas em estatura que ocorrem durante esses períodos. Para testar essa hipótese apropriadamente seria necessário realizar um ensaio clínico randomizado nesse grupo de pacientes. No entanto, algumas evidências demonstram que é possível melhorar o estado nutricional e o IMC de pacientes com doenças crônicas por intervenção dietoterápica, como relatado em estudos realizados em pacientes com insuficiência renal crônica $^{(35)}$, fibrose cística ${ }^{(36)}$ e doença de Crohn ${ }^{(37)}$.

Considerando-se a significativa prevalência de anormalidades nutricionais em pacientes com AIJ e a relação da baixa estatura com a duração e subtipo da doença e com o VSG, a importância do controle estrito e precoce do processo inflamatório nesses pacientes é destacada no presente estudo.

\section{AGRADECIMENTOS}

Agradecemos o valioso auxílio prestado por Ilóite Scheibel, Maria Mercedes Picarelli, Mauro W. Keiserman, Márcia Regina Vítolo, Claiton V. Brenol e Charles L. Kohem.

9. Chaud DM, Hil Rio MO, Yanaguibashi G, Amancio OM: Avaliações dietética e antropométrica em pacientes com artrite reumatóide juvenil [Dietetic and anthropometric assessment in juvenile rheumatoid arthritis patients]. Rev Assoc Med Bras 49: 181-4, 2003

10. Miller ML, Chacko JA, Young EA: Dietary deficiencies in children with juvenile rheumatoid arthritis. Arthritis Care Res 2: 22-4, 1989.

11. Henderson CJ, Lovell DJ, Gregg DJ: A nutritional screening test for use in children and adolescents with juvenile rheumatoid arthritis. J Rheumatol 19: 1276-81, 1992.

12. Zak M, Muller J, Karup Pedersen F: Final height, armspan, subischial leg length and body proportions in juvenile chronic arthritis. A longterm follow-up study. Horm Res 52: 80-5, 1999.

13. Kanamori Y, Miyamoto K, Hosoe H, Fujitsuka H, Tatematsu N, Shimizu K: Transoral approach using the mandibular osteotomy for atlantoaxial vertical subluxation in juvenile rheumatoid arthritis associated with mandibular micrognathia. J Spinal Disord Tech 16: 221-4, 2003

14. Liphaus B, Campos L, Silva C, Carrasa F, Rodrigues M: Avaliação endoscópica de crianças e adolescentes com artrite reumatóide juvenil em uso de antiinflamatórios não hormonais (AINH) [Endoscopic evaluation of children and adolescents with juvenile rheumatoid arthritis using nonsteroidal anti-inflammatory drugs (NSAID)]. Rev Bras Reumatol 41: 332-6, 2001.

15. Ostrov BE: Nutrition and pediatric rheumatic diseases. Hypothesis: cytokines modulate nutritional abnormalities in rheumatic diseases. J Rheumatol 33 Suppl:49-53, 1992. 
16. Klepper SE, Giannini MJ: Physical conditioning in children with arthritis: Assessment and guidelines for exercise prescription. Arthritis Care Res 7: 226-36, 1994.

17. Hillman L, Cassidy JT, Johnson L, Lee D, Allen SH: Vitamin D metabolism and bone mineralization in children with juvenile rheumatoid arthritis. J Pediatr 124: 910-6, 1994.

18. Isaksson OG, Eden S, Jansson JO: Mode of action of pituitary growth hormone on target cells. Annu Rev Physiol 47: 483-99, 1985.

19. Aitman TJ, Palmer RG, Loftus J, et al: Serum IGF-I levels and growth failure in juvenile chronic arthritis. Clin Exp Rheumatol 7: 557-61, 1989

20. Saha MT, Verronen P, Laippala P, Lenko HL: Growth of prepubertal children with juvenile chronic arthritis. Acta Paediatr 88: 724-8, 1999.

21. Wang SJ, Yang YH, Lin YT, Yang CM, Chiang BL: Attained adult height in juvenile rheumatoid arthritis with or without corticosteroid treatment. Clin Rheumatol 21: 363-8, 2002.

22. Haugen MA, Lien G, Flato B, et al: Minor impact of juvenile arthritis on nutritional status in young adult patients. Arthritis Rheum 47: 623-9, 2002.

23. Petty RE, Southwood TR, Baum J, et al: Revision of the proposed classification criteria for juvenile idiopathic arthritis: Durban, 1997. J Rheumatol 25: 1991-4, 1998.

24. World Health Organization. Physical status: the use and interpretation of anthropometry. Report of a WHO Expert Committee. Geneva: World Health Organization; (WHO Technical Report Series, no. 854), 1995.

25. National Center for Health Statistics. NCHS growth curves for children, birth-18 years, United States. Rockville: National Center for Health Statistics; DHEW publication no. (PHS)78-1650. (Vital and health statistics; series 11, no. 165), 1977.

26. National Center for Health Statistics. Anthropometric reference data and prevalence of overweight, United States, 1976-80. Hyattsville: National Center for Health Statistics; DHHS publication no. (PHS) 87-1688. (Vital and health statistics; series 11; no. 238), 1987.
27. Miles LE, Lipschitz DA, Bieber CP, Cook JD: Measurement of serum ferritin by a 2 -site immunoradiometric assay. Anal Biochem 61: 209-24, 1974.

28. Dean AG, Dean JA, Coulombier D, et al: Epi Info, Version 6: A Word Processing, Database, and Statistics Program for Epidemiology on Microcomputers. Atlanta: Center for Disease Control and Prevention, 1994.

29. Norusis MJ: SPSS 11.0 Guide to Data Analysis. Upper Sadlle River: Prentice Hall, 2002.

30. Cottrell A: Gretl Manual: Gnu Regression, Econometrics and Timeseries Library. Department of Economics, Wake Forest University; 2004. http://gretl.sourceforge.net/manual.pdf (accessed 18 July 2005).

31. Greenland S: Modeling and variable selection in epidemiologic analysis.Am J Public Health 79: 340-9, 1989.

32. Harrell FE Jr, Lee KL, Matchar DB, Reichert TA: Regression models for prognostic prediction: advantages, problems, and suggested solutions. Cancer Treat Rep 69: 1071-77, 1985.

33. Walton AG, Welbury RR, Thomason JM, Foster HE: Oral health and juvenile idiopathic arthritis: a review. Rheumatology (Oxford) 39: 550-5, 2000.

34. Di Toro R, Polito C: Nutrition in juvenile rheumatoid arthritis. Nutr Res 17: 741-58, 1997.

35. Simmons JM, Wilson CJ, Potter DE, Holliday MA: Relation of calorie deficiency to growth failure in children on hemodialysis and the growth response to calorie supplementation. N Engl J Med 285: 653-6. 1971.

36. Shepherd RW, Holt TL, Thomas BJ, et al: Nutritional rehabilitation in cystic fibrosis: controlled studies of effects on nutritional growth retardation, body protein turnover, and course of pulmonary disease. J Pediatr 109: 788-94, 1986.

37. Kirschner BS, Klich JR, Kalman SS, DeFavaro MV, Rosenberg IH: Reversal of growth retardation in Crohn's disease with therapy emphasizing oral nutritional restitution. Gastroenterology 80: 10-5, 1981. 